\title{
Neuromuscular function in limb girdle dystrophy
}

\author{
AY BELANGER, ${ }^{*}$ AJ McCOMAS \\ From the Departments of Neurosciences and Medicine, McMaster University Medical Centre, Hamilton, \\ Ontario, Canada
}

SUMMARY The contractile properties of ankle dorsiflexor and plantarflexor muscles in 20 patients with limb girdle muscular dystrophy have been compared with those in matched controls. Twitch and voluntary torques were significantly smaller in the patient population and in nine patients it was impossible to record a twitch from tibialis anterior, a dorsiflexor muscle studied in detail. The disease process evidently ran a more rapid course in tibialis anterior than in plantarflexor muscles and this susceptibility was related to some aspect of the muscle other than its fibre type composition. Surviving fibres in dorsiflexor and plantarflexor muscles did not reveal evidence of excitation-contraction uncoupling; they exhibited normal post-activation potentiation and fatigue properties. Some patients were initially incapable of exciting their motor units maximally during voluntary contractions. A finding of possible pathogenetic significance was that one patient, with prominent calves, developed exceptionally large voluntary torque in his plantarflexor muscles.

Relatively little is known concerning the contractile properties of muscles in patients with limb girdle muscular dystrophy and the information available has come from two intrinsic muscles of the hand and foot, the adductor pollicis ${ }^{1-3}$ and the extensor digitorum brevis. ${ }^{4}$ Since the weakness in this disorder is most evident in larger, more proximal, muscles than in smaller distal ones, there is added interest in studying the former. Accordingly, in the present study measurements of voluntary and evoked contractions have been made in the dorsiflexor and plantarflexor muscles of the ankle, together with the associated muscle impulse activity. As in an earlier study of myotonic dystrophy, ${ }^{5}$ we have sought information concerning certain fundamental aspects of contractile behaviour in limb girdle muscular dystrophy; these are the relative involvement of fasttwitch and slow-twitch muscle fibres, the relationship between force development and evoked muscle impulse activity, the ability of patients to utilise fully their available muscle mass and the potentiating and fatigue properties of the dystrophic muscle fibres.

Address for reprint requests: Dr A J McComas, Room 4U7 McMaster University Medical Centre, P.O. Box 2000, Hamilton, Ontario, Canada L8N $3 Z 5$

*Present address: Ecole de réadaptation, Université Laval, Québec, Canada.

\section{Methods}

\section{Subjects and patients}

Experiments were performed on 20 patients with limb girdle muscular dystrophy (13 males and 7 females aged 19 to 63 years) and on the same number of healthy subjects; the latter were closely matched to the patients for age, sex, weight and height. The diagnosis of limb girdle muscular dystrophy was made in all cases on the basis of the clinical features and EMG; in addition, muscle biopsies had been carried out on some patients and there was sometimes a history of an affected relative. All patients and subjects volunteered for the experiments and were paid. The project carried the approval of the Ethics Committee at McMaster University.

\section{Techniques and protocol}

The study was carried out on the dorsiflexor and plantarflexor muscles of the ankle; the tibialis anterior was the dorsiflexor muscle used for analysis of the twitch. The person sat with one leg mounted in a torque measuring device, ${ }^{6}$ the hip and knee being at right-angles. The ankle was adjusted to either $20^{\circ}$ of plantarflexion or to $10^{\circ}$ of dorsiflexion, depending on whether the dorsiflexor or plantarflexor muscles respectively were to be examined. In six patients passive dorsiflexion was restricted due to contracture of the Achilles tendons. The arrangements of the stimulating and recording electrodes were identical to those described previously, ${ }^{7}$ and all measurements of evoked activity were made with stimuli of supramaximal intensity applied to the respective motor nerve fibres. The leg was warmed with an infrared lamp so as to maintain the skin temperature of $34^{\circ}-38^{\circ} \mathrm{C}$. After the "resting" twitches and $\mathrm{M}$-waves had been recorded, post-activation potentia-

Received 25 May 1984 and in revised form 6 November 1984 Accepted 10 November 1984 
Table Summary of electrical and mechanical properties of muscles in controls and patients with limb girdle muscular dystrophy (LGMD)

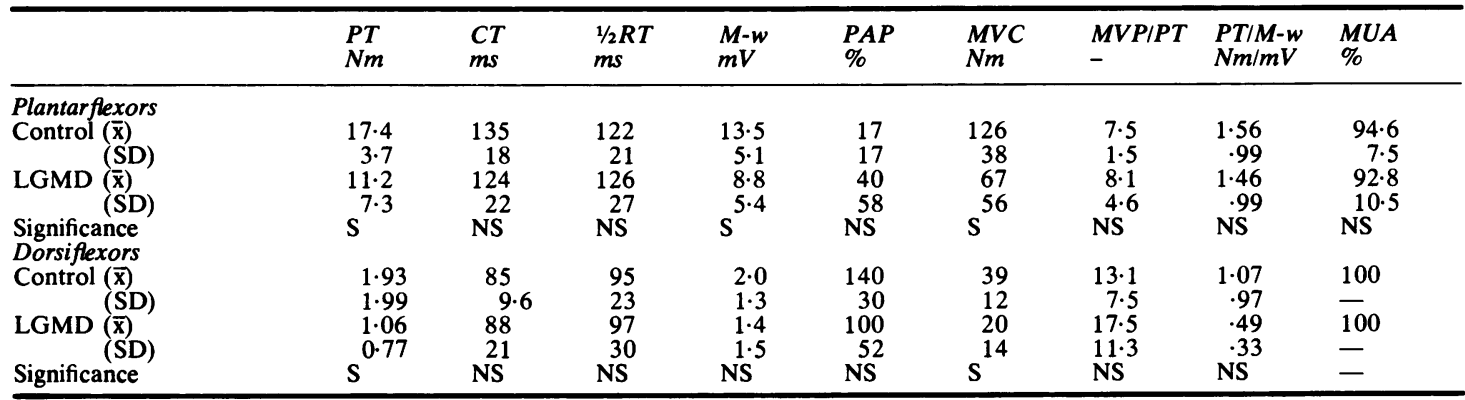

$P T$, twitch torque; $C T$, contraction time; $1 / 2 R T$, half-relaxation time; $M-w, M$-wave; $P A P$, postactivation potentiation; $M V C$, torque during maximum voluntary contraction; $M U A$, motor unit activation.

tion was assessed following $5 \mathrm{~s}$ of maximal voluntary contraction. Maximal voluntary torque was then measured, each subject being allowed several attempts; the degree of motor unit activation was analysed by the interpolated twitch technique. ${ }^{8}$ The time-course of fatigue was examined by monitoring the loss of voluntary torque during 60 seconds of maximal effort; the site of fatigue was investigated by comparing the maximal evoked twitch and $\mathrm{M}$-wave immediately after the fatiguing contraction.

All measurements were made from Polaroid photographs of stored displays on oscilloscopes. Correlation analyses were performed using the Pearson coefficient of correlation. Significance between means was estimated with the Student $t$ test at the $5 \%$ level; corrections for non-homogeneity of variance were undertaken. ${ }^{9}$

\section{Results}

\section{Twitch properties}

As expected, twitch torques were reduced in the tibialis anterior and plantarflexor muscles of patients with limb girdle muscular dystrophy, although not to equal extents. Thus, the mean twitch torque for tibialis anterior was only $54.9 \%$ of the control mean whereas that of plantarflexors was slightly higher $(64.4 \%$; table). In the case of tibialis anterior, there were nine patients in whom it was not possible to record a contraction, while the remaining 10 patients generated torques within the normal range (fig 1). The findings for plantarflexors were different in that, although there was only one patient with an undetectable twitch, there were nine in whom the torque values fell below those obtained in controls. The twitch speeds of the normal and dystrophic muscles were similar, neither tibialis anterior nor plantarflexors exhibiting significant differences in mean contraction and half-relaxation times between the groups of subjects (table; fig 1). Unlike the findings in myotonic dystrophy there was no evidence of a relationship between twitch torque and contraction time.
Evidence of post-activation potentiation was sought by eliciting twitches after brief voluntary contractions (see Methods). In the tibialis anterior muscles of patients the mean twitch torque doubled, and this degree of potentiation was not significantly less

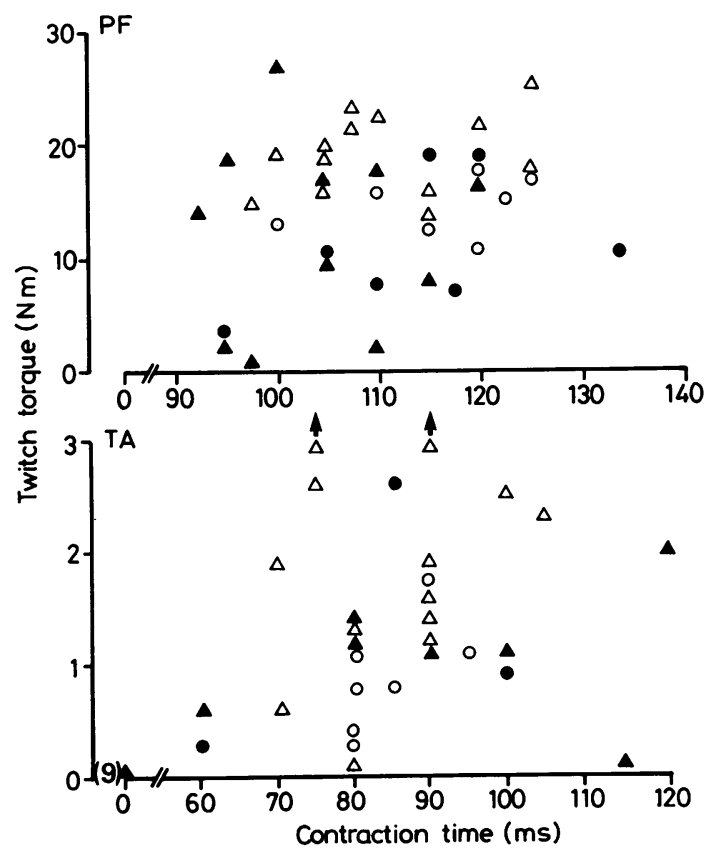

Fig 1 Twitch torque as a function of contraction time for plantarflexor and tibialis anterior muscles. Values for males and females are shown as triangles and circles respectively. Open symbols denote the control values while filled symbols identify results from patients with limb girdle muscular dystrophy. Arrows indicate values exceeding limits of axes. Note the absent plantarflexor twitch in one patient and the absent tibialis anterior twitches in nine patients wth limb girdle muscular dystrophy. 


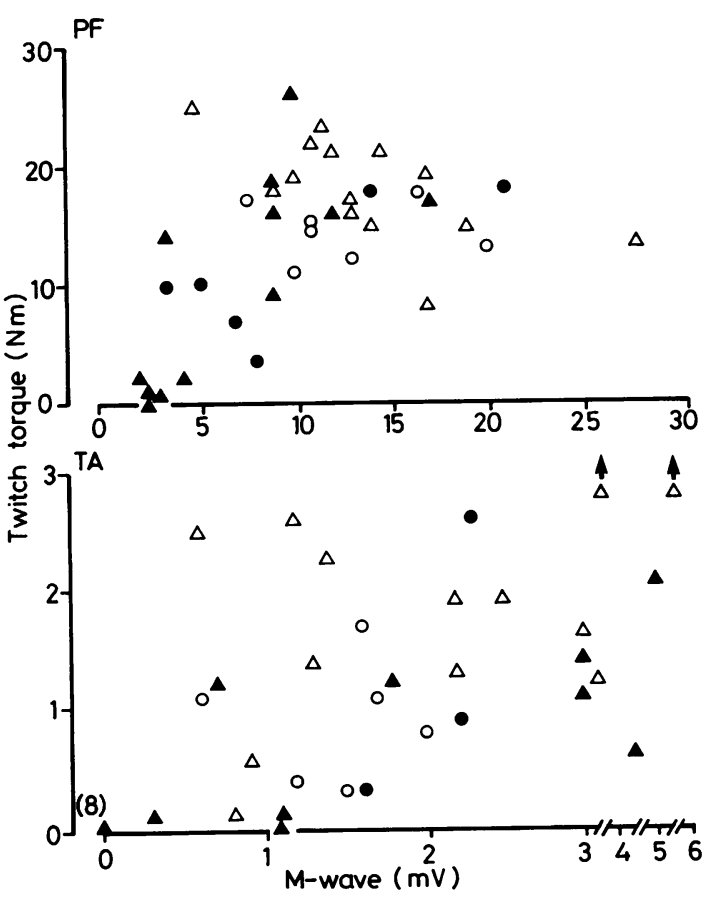

Fig 2 Twitch torque as a function of the muscle compound action potential amplitudes ( $M$-wave) in plantarflexor and tibialis anterior muscles. Values for males and females are shown as triangles and circles respectively. Open symbols denote control values while filled symbols identify results from patients. Arrows indicate values exceeding limits of axes. Note the considerable scatter of results from dystrophic tibialis anterior muscles and the absence of tibialis anterior muscles and the absence of tibialis anterior twitches and $M$-waves in 8 patients.

than that of control muscles (table). Plantarflexor muscles normally show less potentiation than tibialis anterior ${ }^{7}$ and this was found to be true for the dystrophic patients also; although there was rather more potentiation in plantarflexor muscles of patients than controls, the large variation between patients rendered the difference statistically insignificant. In all the muscles tested the contraction and half-relaxation times of the potentiated twitches became shorter, irrespective of the extent of the potentiation; this finding indicated that potentiation had resulted from an increase in the intensity, rather than in the duration, of the active state..$^{10}$

The question of efficacy of muscle impulse activity in eliciting contractile responses was assessed by comparing the $\mathrm{M}$-wave amplitudes and twitch torques (fig 2). In the dystrophic group, a significant correlation was obtained only for the plantarflexor muscles $(r=0.59$; tibialis anterior $r=0 \cdot 45)$. For plantarflexor muscles, one patient showed a small M-wave $(2.5 \mathrm{mV})$ but no detectable twitch torque. For tibialis anterior muscles, twitches and M-waves were not detectable in eight patients, while in another patient a small M-wave $(1.2 \mathrm{mV})$ was not associated with any twitch torque (fig 2). Despite such cases, however, the twitch: $M$-wave ratios were not significantly different from the normal values in the dystrophic tibialis anterior and plantarflexor muscles (table).

\section{Voluntary contractions}

The measurement of maximal isometric voluntary contraction was made following at least three attempts, each lasting approximately 4 seconds. Figure 3 shows plantarflexor and dorsiflexor voluntary torques plotted against each other. In keeping with earlier findings in normal subjects' the male controls generated greater torques than their female counterparts. As expected, limb girdle muscular dystrophy patients were weaker than their matched controls for both plantarflexor and dorsiflexor muscles (table); nevertheless, approximately one-third of patients had normal torque values. An important observation was that the two muscle groups could be affected differently in each patient; for example, one patient showed relatively strong plantarflexor muscles $(55 \mathrm{Nm})$ but weak dorsiflexor muscles $(2 \mathrm{Nm})$. Another patient presented with weaker plantarflexor than dorsiflexor muscles while in another patient no dorsiflexor torque could be recorded although $15 \mathrm{Nm}$ of torque was generated by plantarflexor muscles. Despite these instances, there was a significant correlation between the maximal torque

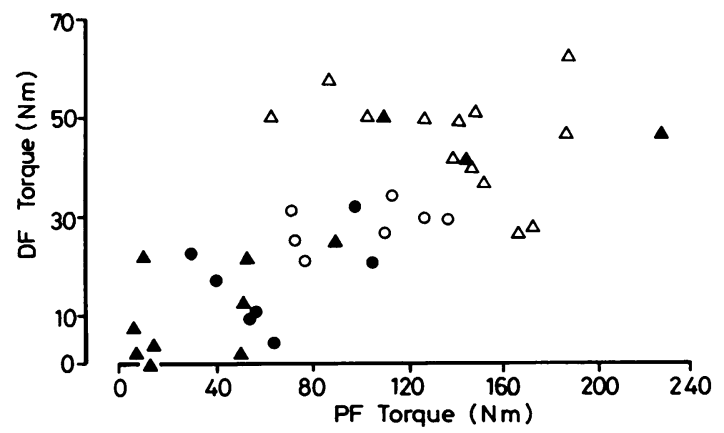

Fig 3 Maximum voluntary dorsiflexor torque as a function of plantarflexor torque in patients with limb girdle muscular dystrophy (filled symbols) and their controls. Values for males and females are shown as triangles and circles respectively. As expected, patients with limb girdle muscular dystrophy were weaker than controls. Note the considerable variability of muscle strength among male dystrophic patients. 
values of plantarflexor and dorsiflexor muscles in the patients $(r=0.76)$ but not in controls $(r=0.21)$. Of interest was the fact that the highest plantarflexor voluntary torque generated during the course of the study was by a 34 year old male patient with limb girdle muscular dystrophy $(235 \mathrm{Nm})$. The voluntary dorsiflexor torques generated by patients with absent tibialis anterior twitches were ascribed to residual activity in the long extensors of the toes. As a result of such presumptive activity, no significant correlation was found between the tibialis anterior twitches and the dorsiflexor voluntary torques in the patient population $(r=0.45)$, while a significant correlation was observed for the control subjects $(r=0 \cdot 60)$. For plantarflexor muscles, both the dystrophic and the control subjects showed significant correlations between twitch and voluntary torque values $(r=0.81$ and 0.76 respectively).

To establish whether or not dystrophic patients were making full use of their surviving motor units during maximal isometric strength testing, we employed the twitch interpolation technique. ${ }^{8}$ Although some patients differed from controls in not being able to develop their greatest voluntary torques at their first or second attempts, the degree of motor unit activation which was ultimately achieved was similar in the two populations of subjects (table). Thus, when the final values were compared, it was found that tibialis anterior activation was complete in all subjects within both populations. Full activation of plantarflexor motor units was less readily attained, in keeping with earlier observations, ${ }^{8}$ but

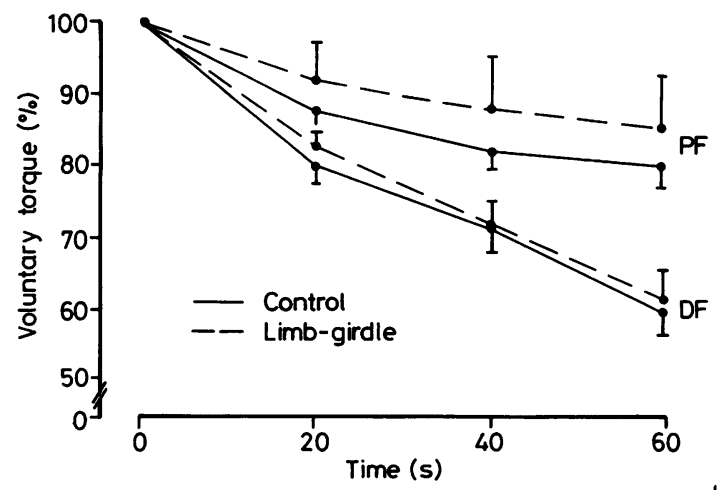

Fig 4 Decline of maximum voluntary torque in dorsiflexor and plantarflexor muscles of patients with limb girdle muscular dystrophy and control subjects. Mean values are shown with their standard errors of the mean. No difference was seen in the fatigue behaviour, as measured by the amplitude and the rate of decline of voluntary tension, between the dystrophic patients and controls; the dorsiflexor muscles showed less resistance to fatigue. the values for controls were not significantly different from those of patients.

\section{Muscle fatigue}

The susceptibility to muscle fatigue in patients with limb girdle muscular dystrophy was assessed by measuring the decline of isometric torque during $60 \mathrm{~s}$ of maximal effort. Maximal stimuli were interpolated at $20 \mathrm{~s}$ intervals to ascertain whether or not there was a decline in voluntary motor unit activation; ${ }^{7}$ in general, the degree of activation remained similar to that at the start of the effort, being complete in tibialis anterior but sometimes less so in plantarflexors. These results showed that the central nervous system did not contribute significantly to the fatigue observed in these experiments.

When the results for the patients and controls were compared (fig 4), it was evident that fatigue was greater in dorsiflexor than in plantarflexor muscles in both groups of subjects; for each type of muscle, however, the mean degree of fatigue was similar in the two subject populations.

Having excluded a central cause for fatigue (see above), the peripheral mechanism was analysed by comparing the $\mathbf{M}$-wave and the twitch torque at the end of the voluntary contraction. Whereas any change in $\mathrm{M}$-wave amplitude was less than the resolution of our recording equipment, the mean tibialis anterior twitch torques declined to $40 \%$ and $55 \%$ of their initial values in the controls and the dystrophic patients respectively. In contrast, the plantarflexor torques were only slightly reduced in both populations after the fatiguing exercise.

\section{Discussion}

Muscle biopsies studied elsewhere" ${ }^{11}$ have indicated preferential involvement of type II fibres by the disease process in limb girdle muscular dystrophy and consequently one might have anticipated slowing of the isometric twitch, as was observed in the extensor hallucis brevis. ${ }^{4}$ The normal twitch speeds found in the present study were therefore unexpected, although similar results had been obtained in the adductor pollicis by Desmedt.' It is possible that the dystrophic process affects twitch speed to different extents in various muscles of the body; however, an equally plausible explanation would be that conclusions based on histochemical studies, none of which have included quantitative data, may have been invalidated by tissue sampling problems. ${ }^{12}$ In limb girdle muscular dystrophy the muscles not only behaved as if they had the correct proportions of fast and slow twitch units but as in myotonic dystrophy, ${ }^{5}$ the surviving fibres did not show any impairment of their potentiating and fatigue properties. The only 
reference which we have been able to find to fatigue properties in limb girdle muscular dystrophy is the report by Blank et al, ${ }^{13}$ which simply showed normal EMG patterns during fatiguing contractions in six patients.

The normal potentiation found in this study does not support the concept of excitation-contraction uncoupling in limb girdle muscular dystrophy put forward by Desmedt ${ }^{1}$ and by Takamori et al ${ }^{3}$ both of whom examined the adductor pollicis. Another observation from the present study which suggests that uncoupling was not important in our patients was the preservation of the normal proportionality between twitch torque and M-wave. However, these negative observations did not exclude the possibility that uncoupling may sometimes occur at a given stage in a dystrophic muscle, for striking twitch torque: M-wave disproportionality was occasionally seen in an earlier study of myotonic dystrophy. ${ }^{5}$

One of the most interesting findings in the present study was the tendency for the twitch torques of the tibialis anterior muscles to be either normal or else negligible; this situation was in contrast to that for plantarflexors where graded reductions in torque were observed in the patient population. One interpretation of the findings would be that the dystrophic process runs a more rapid course in tibialis anterior than in plantarflexors. Further, the absence of measureable tibialis anterior torque in some patients furnishes conclusive proof that the dystrophic process can destroy almost the entire populations of type I and type II fibres in a given muscle. Since plantarflexor twitches were nearly always present in those patients with absent tibialis anterior responses, it follows that type 1 fibres may still be present in one muscle (the triceps surae complex; $70-100 \%$ type 1 fibres) while totally destroyed in another (tibialis anterior; $50-70 \%$ type 1 fibres). It therefore appears that, in limb girdle muscular dystrophy as in myotonic dystrophy, ${ }^{5}$ fibre type composition is not the factor which determines whether or not the dystrophic process affects some muscles more severely than others. A further resemblance to myotonic dystrophy ${ }^{5}$ was the inability of most limb girdle muscular dystrophy patients to develop maximal voluntary dorsiflexor or plantarflexor torque at the initial attempt; instead several repetitions were required before their maximum strength could be realised. The fact that motor unit activity could be increased by repeated voluntary effort during one experimental session indicated that the failure to achieve greater muscle strength was not a consequence of any structural changes in the descending motor pathways or in their synaptic connections. It also indicated that, in patients with moderately severe muscle weakness, the relatively few move- ments attempted during the day may well render motoneurons transiently incapable of sudden maximal activation. This conclusion is in keeping with that reached in normal individuals subjected to limb immobilisation. ${ }^{14}$ is The observation that some dystrophic patients generated greater muscle strength after repeated trials has therapeutic implications, for it suggests the possibility of better muscular performance through the adoption of appropriate exercise programmes. It also suggests that muscle strength in patients with muscular dystrophy may sometimes be underestimated, unless several attempts are made by the patient during strength testing.

There is one last observation which, although derived from a single patient, deserves comment; this was the exceptionally large maximal voluntary plantarflexor torque developed by a 34-year-old man with hypertrophied calf muscles. Such a result clearly implies that muscle enlargement in dystrophy may sometimes reflect an increase in excitable muscle mass rather than hyperplasia of connective tissue. Although anecdotal in nature, we have nevertheless been impressed by comments made by adult patients with either limb girdle muscular dystrophy, facioscapulohumeral muscular dystrophy or spinal muscular atrophy to the effect that, in their youth, they were unusually strong or else excelled in athletics. The concept of unusual muscle strength in early muscular dystrophy originated with Duchene ${ }^{16}$ and would be consistent with reports of muscle fibre hypertrophy, without degeneration, in some patients; ${ }^{17} 18$ if valid, it throws new light on the pathogenesis of dystrophy.

We are indebted to the Muscular Dystrophy Association of Canada for a grant-in-aid to Dr Alan J. McComas and for a pre-doctoral fellowship to Alain Y. Belanger. Valuable assistance was received from Nancy Kowalchuk, Judy Moffatt and Glenn Shine.

\section{References}

${ }^{1}$ Desmedt JE. The isometric twitch of human muscle in the normal and dystrophic states. In: Milhorat, AT, ed. Exploratory Concepts in Muscular Dystrophy and Related Disorders. New York: Excerpta Medica 1967: 224-30.

${ }^{2}$ Takamori M. Contractility and supersensitivity to adrenaline in dystrophic muscle. J Neurol Neurosurg Psychiatry 1975;38:483-92.

${ }^{3}$ Takamori M, Hazama $R$, Tsujihata $M$. Active state properties of denervated and immobilized muscle: comparison with dystrophic muscle. Neurol (NY) 1978;28:603-8.

${ }^{4}$ Sica REP, McComas AJ. An electrophysiological investigation of limbgirdle and facioscapulohumeral dys- 
trophy. J Neurol Neurosurg Psychiatry 1971;34:46974.

${ }^{5}$ Belanger AY, McComas AJ. Contractile properties of muscles in myotonic dystrophy. J Neurol Neurosurg Psychiatry 1983;46:625-31.

- Marsh E, Sale D, McComas AJ, Quinlan J. Influence of joint position on ankle dorsiflexion in humans. J Appl Physiol 1982;52:1636-42.

${ }^{7}$ Belanger AY, McComas AJ, Elder GBC. Physiological properties of two antagonistic human muscle groups. Eur J Appl Physiol 1983;51:381-93.

${ }^{8}$ Belanger AY, McComas AJ. Extent of motor unit activation during effort. J Appl Physiol 1981;51:1131-5.

" Snedecor GW, Cochran WG. Statistical Methods. Iowa Univ. State Press, 1974.

${ }^{10}$ Desmedt JE, Hainaut K. Kinetics of myofilament activation in potentiated contraction: staircase phenomenon in human skeletal muscle. Nature;217:529-32.

"Dubowitz V, Brooke MH. Muscle Biopsy: A Modern Approach. London: WB Saunders 1973.

12 Elder GBC, Bradbury K, Roberts R. Variability of fiber type distributions within human muscles. J Appl
Physiol 1982;53:1473-80.

${ }^{13}$ Blank A, Gonen A, Magora A. The electrophysiologic pattern of development of muscular fatigue in muscular dystrophy. Electromyogr Clin Neurophysiol 1980; 20: 3-18.

${ }^{14}$ Fuglsang-Frederiksen A, Scheel U. Transient decrease in number of motor units after immobilization in man. J Neurol Neurosurg Psychiatry 1978;41:924-9.

is Sale D, McComas AJ, MacDougall JD, Upton ARM. Neuromuscular adaptation in human thenar muscles following strength training and immobilization.J Appl Physiol 1982;53:419-24.

${ }^{16}$ Duchenne GB. De l'Electrisation Localisée et son Application da la Pathologie et à la Therapeutique, 2nd ed. Paris: Baillière et fils 1861.

${ }^{17}$ Erb W. Dystrophia muscularis progressiva; Klinische und pathologischanatomische Studien. Deut $Z$ für Nerven 1891;1:173-261.

18 Walton JN, Gardner-Medwin D. Second thoughts on classification of the muscular dystrophies. In: Current Research in Muscular Dystrophy. Proceedings of the Fourth Symposium. London: Pitman Medical 1968. 